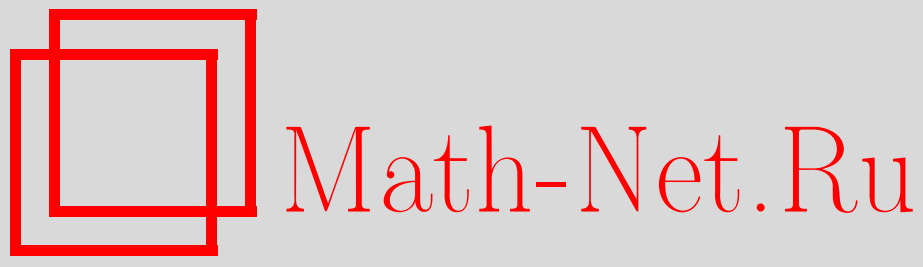

А. М. Вершик, Двойственность Крейна, позитивные 2-алгебры и дилатация коумножений, Функи. анализ и его прил., 2007, том 41, выпуск 2, 24-43

DOI: https://doi.org/10.4213/faa2857

Использование Общероссийского математического портала MathNet.Ru подразумевает, что вы прочитали и согласны с пользовательским соглашением http://www. mathnet.ru/rus/agreement

Параметры загрузки:

IP: 3.91 .87 .62

26 апреля 2023 г., 12:59:07

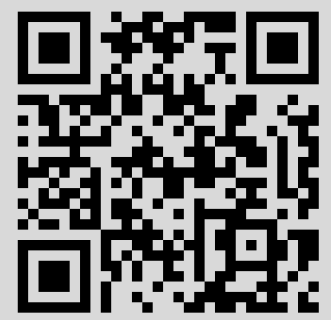




\title{
Двойственность Крейна, позитивные 2-алгебры и дилатация коумножений*
}

\author{
(c) 2007. А. М. ВЕРшик
}

К столетию Марка Григорьевича Крейна

\section{§. Введение}

Цель этой работы - постановка проблемы о подъеме инволютивных алгебр с положительными умножением и коумножением (мы называем их позитивными 2-алгебрами) в инволютивную биалгебру, например в групповую биалгебру конечной группы или инверсной полугруппы. Эта задача возникла в связи с проблемами, относящимися к теории ассоциативных схем, а также в связи с некоторыми комбинаторными вопросами теории представлений. Ее можно также трактовать как задачу о дилатации операторов умножения и коумножения и, таким образом, связать с теорией дилатаций полугрупп операторов, которой занимался М. Г. Крейн. Мы называем ее кратко задачей о дилатации коумножений или о дерандомизации случайного умножения.

Многие идеи в этой области берут начало в работах М. Г. Крейна о двойственности и положительности; поэтому мы начинаем статью (§2) с краткого исторического очерка о положительных умножениях и двойственности, показывающего, каким сложным путем идеи Крейна о двойственности и положительности алгебр, наконец, объединились в наше время. Этот очерк неполон и не затрагивает многих смежных вопросов (см. [4]).

Наиболее яркий конкретный пример нашей основной проблемы дан в $\S 3,-$ это вопрос об алгебре Гекке $H_{n}(q)$ и ее вложении в $G L(n, \mathbb{C})$, рассмотренный с разных точек зрения.

В §4 мы определяем категорию позитивных инволютивных 2-алгебр, т. е. пространств с положительными (относительно заданных инволюции и коинволюции) умножением и коумножением, называемых далее позитивными 2-алгебрами. Категория таких 2-алгебр включает категории биалгебр, алгебр Хопфа и гипергрупп: умножение и коумножение здесь не гомоморфизмы, а только положительные отображения. Функториально она эквивалентна категории *-алгебр в положительной двойственности, введенной автором в 1971 г. в [5], но в этой работе мы используем язык биалгебр и умножений-коумножений вместо языка алгебр в двойственности. Подчеркнем, что сама идея двойственности является в известном смысле продолжением идей М. Г. Крейна. Проблема подъема положительных операций до мультипликативных традиционна для функционального анализа и его приложений [18]. Здесь мы формулируем ее в чисто алгебраическом контексте. Определяются два вида подобъектов в инволютивных биалгебрах (§5), а затем в 66 дается точная постановка общей задачи о том,

*Частично поддержано грантами РФФИ 05-01-00899 и CRDF RUM1-2622-ST-04. 
что значит вложить позитивную 2-алгебру в биалгебру. В разд. 6.4 мы подробно разбираем самый простой (двумерный бикоммутативный) пример - классифицируем двумерные алгебры Гекке как позитивные инволютивные 2-алгебры - и иллюстрируем на этом примере различие между двумя типами дилатаций. Общая проблема о дилатациях позитивных 2-алгебр даже в размерности 3 далека от своего решения.

\section{§2. Работы М. Г. Крейна о двойственности и положительности в алгебрах}

В 40-х годах М. Г. Крейн занимался теорией представлений и опубликовал несколько статей на эту тему. Статьи [15], [16] посвящены инвариантным эрмитово-положительным ядрам на однородных пространствах и примыкают к излюбленной им тематике о положительной определенности и ее связях с теорией операторов ${ }^{1)}$. Эти работы не были, насколько мне известно, продолжены им, но получили достаточную известность. В частности, один из результатов статьи [16] - теорема об эквивалентности алгебраической и скалярной положительности - понадобится нам далее. В довоенной статье [13] и более полной статье на ту же тему [14], которые внешне никак не связаны со статьями [15], [16] (хотя в [14] приведен основной результат из [16]), популярная тогда двойственность Понтрягина-Ван Кампена для локально компактных абелевых групп была перенесена на компактные неабелевы группы (несколько ранее это было сделано, правда, в меньшей общности, Т. Таннакой, о чем Крейн узнал от Д. А. Райкова после публикации работы [14]).

Если в абелевом случае двойственность не выводит за пределы категории абелевых групп - «функтор Фурье» сопоставляет данной группе группу ее характеров и обобщает классическую теорию преобразования Фурье с групп $\mathbb{Z}$ или $\mathbb{R}$ на все локально компактные абелевы группы, то в неабелевом случае двойственный объект есть не группа, а некоторая алгебра. То, что называют сейчас блок-алгеброй Крейна [15], есть коммутативная алгебра, разбитая в не более чем счетную сумму простых конечномерных алгебр над $\mathbb{C}$ с выделенным базисом. Этот базис есть базис матричных единиц во всех классах эквивалентных неприводимых представлений исходной группы. Точнее, если $G$ - компактная группа, то нетрудно увидеть в двойственной к ней по Крейну блок-алгебре алгебру всех непрерывных функций на этой группе, снабженную разложением в прямую сумму минимальных двусторонних идеалов и базисом из матричных элементов всех унитарных неприводимых представлений группы $G$. Теорема двойственности Крейна состоит в том, что эта блок-алгебра определяет группу с точностью до изоморфизма как группу мультипликативных на базисе скалярных гомоморфизмов. Доказательство эффектно использует теорию нормированных колец Гельфанда ${ }^{2)}$. Крейн реализует групповую алгебру компактной группы как, по его выражению, блок-алгебру, т. е. как алгебру функций на счетном множестве классов неприводимых унитарных представлений этой группы с

1) «Слова «положительно определенная функция» действуют на меня, как звук трубы на боевого коня», - в присущем ему приподнятом стиле сказал Марк Григорьевич в своем докладе на конференции по функциональному анализу в 1958 г. в Одессе.

2) Рискну предположить, что успехи только что появившейся тогда гельфандовской теории нормированных колец и особенно теории бесконечномерных представлений и стимулировали занятия этими вопросами и М. Г. Крейна, и А. Н. Колмогорова (см. переписку в [12]). 
матричными значениями; выбирая определенным образом базис в этой алгебре, мы приходим к теореме двойственности.

Более симметричная формулировка теорем Крейна возникает, если освободиться от базисов и использовать инвариантный язык теории двойственности алгебр как векторных пространств, см. §5. Первая алгебра есть групповая алгебра группы $G$ над некоторым полем, а двойственная алгебра есть алгебра непрерывных функций на этой группе со значениями в том же поле. Такую формулировку в духе бурбакистской теории двойственности векторных пространств [1] предложил автор в работе [5], где вводилась категория пар *-алгебр в положительной двойственности и ее геометрический вариант - категория пакетов (выпуклых компактов специального вида). Эта теория была развита в дальнейшем С. В. Керовым в [9], [10] и недавно продолжена в [6], [3], [7].

Современная формулировка такой двойственности использует терминологию биалгебр и алгебр Хопфа. Она заключается в том, что в групповой алгебре есть коммутативное коумножение, а в коммутативной алгебре функций на группе есть сверточное коумножение и каждая из этих двух биалгебр определяет другую. Эта формулировка без всяких дополнений годится для конечных групп; позже она была обобщена на универсальные обертывающие алгебры групп Ли. Но в общем случае локально компактных групп она требует серьезных топологических комментариев, и в работе Крейна, собственно, и приводились те соображения из теории нормированных колец, которых достаточно для компактных групп. Двойственность для общих локально компактных групп в продолжение работ Таннаки-Крейна рассматривали и многие другие. Теория двойственности групповых алгебр и алгебр функций на группе привела в конце концов, правда, не столько по внутренним причинам, к теории квантовых групп. Кроме того, эти работы стали исходными для теории категорий Таннаки-Крейна в алгебраической геометрии [22], [26], двойственности Таннаки-Крейна для группоидов, понятия моноидальных категорий (см. [25], [30], [23]), теории гипергрупп и многозначных групп, теории обобщенного сдвига, некоммутативного интегрирования (И. Сигал, В. Стайнспринг, Г. И. Кац и др.) Всему этому посвящены не одна сотня работ и несколько десятков книг.

И все же следует заметить, что сама по себе двойственность для некоммутативных групп в абстрактной форме, если она не привлекает каких-либо специальных свойств группы, тавтологична и, не побоимся сказать, не имела серьезных аналитических последствий, сравнимых с теорией Фурье для абелевых групп. Причина состоит в том, что категорию, двойственную к категории групповых алгебр, трудно описать независимо, в инвариантных терминах, даже в конечномерном случае. Для содержательного обобщения теории Фурье и построения гармонического анализа требуется дополнительная структура на группе или алгебре, фиксирующая какую-либо инвариантно определенную коммутативную подгруппу (подалгебру) - например, картановскую подгруппу (в теории полупростых алгебр Ли), алгебру Гельфанда-Цетлина (в теории симметрических или подобных ей групп) и т. д. Тогда двойственность становится аналитическим инструментом для изучения групп или алгебр.

В то же время истинное развитие идей двойственности М. Г. Крейна пошло совсем в другом направлении, в котором идеи двойственности объединились 
с теоремами положительности из работ [15], [16]. А именно, реальное и, повидимому, не вполне предусмотренное авторами ${ }^{1)}$ приложение открылось гораздо позже, с одной стороны, в теории двойственности алгебр (см. выше), а с другой, с большим запозданием, в алгебраической комбинаторике и теории ассоциативных схем (см., например, [2]). Именно там понятие блок-алгебры (алгебры Крейна) стало образцом для обобщений и использовалось уже не только для групповых алгебр. Говоря современным языком, состоялся переход от рассмотрения биалгебр и алгебр Хопфа к рассмотрению систем (2-алгебр) с менее жестким согласованием умножений и коумножсений. И решающим аргументом в построении соответствующей теории стала как раз одна из теорем работы [16] о положительности, а не сама по себе двойственность.

Запутанная линия ссылок на эти работы Крейна вкратце выглядит так. Условие положительности для очень специального случая было введено Д. Хигманом в 1964 г.; затем Л. Скотт опубликовал коротенькое замечание в «Notices» [27] о том, что его коллега и специалист по гармоническому анализу С. Дункл обратил его внимание на то, что в работе Крейна [15] есть общее условие положительности для однородных пространств и поэтому условие Хигмана, как пошутил Скотт, является новым только для рассмотренного Хигманом примера. За этим последовали работы Хигмана [24], Скотта [28] и многих других по алгебраической теории ассоциативных схем (см. [2]), после которых условие положительности констант Крейна стало общепринятым и использовалось постоянно. Интересно, что все эти работы, как и последующие работы по этой тематике, тяготеют к работе [14] о блок-алгебрах и именно блок-алгебры обобщаются в них на негрупповую ситуацию (которой не было у Крейна), но реально в этих работах используются не столько результаты из [14] о построении блок-алгебры компактной группы, сколько простое, но ключевое для всех этих рассмотрений условие положительности функций для однородных пространств из работ [16], [14]. Заметим, что предварительная статья 1941 г. [13] была уже во время войны замечена Бохнером [19], и, видимо, поэтому основной результат работы [13] стал тогда же известен на Западе, во всяком случае специалистам по функциональному анализу. Любопытно еще и то, что эти работы Крейна заметили и стали использовать, с почти тридцатилетним запозданием, в своих исследованиях по алгебраической комбинаторике математики, работавшие в США и Японии, а не на Украине или в Москве, где было немало специалистов, занимавшихся ровно теми же вопросами, и где работы Крейна, казалось бы, должны были быть более известными. Впрочем, при подготовке упомянутой выше работы [5], где введена так называемая положительная двойственность *-алгебр и постулированы положительность умножения и коумножения, я также в качестве образца имел в виду скорее теоремы Крейна-Таннаки и его последователей о двойственности, нежели известные мне тогда работы [15], [16], [14]. Работа [5] о положительных спариваниях *-алгебр и была задумана как попытка обобщения двойственности Крейна с групп на однородные пространства двойных классов, но затем выяснилось, что введенное ослабление требования

1)В 1980 г. Марк Григорьевич, показывая мне присланную ему автором (кажется, Ф. Дельсартом) книжку по алгебраическому кодированию и схемам отношений, где его имя появлялось через страницу, говорил с некоторым смущением: «Я тут ни при чем и совершенно этого не понимаю». «Это» и была двойственность Крейна, только не для групповых алгебр компактных групп, как у него, а для алгебр, порожденных ассоциативными схемами. 
мультипликативности охватывает гораздо более общие объекты, не обязательно групповой природы.

Но в этой работе мы соединяем основную тему и с другим направлением, которым также занимался М. Г. Крейн - теорией дилатаций; эта связка, по-видимому, является новой. В функциональном анализе теория дилатаций представлена работами Фойяша-Надя, школы Крейна (дилатация сжатий), В. Стайнспринга (теорема о дилатации вполне положительных отображений), M. А. Наймарка (подъем положительных операторных мер до проекторных или спектральных мер) и др.; теперь она появляется и в задачах чисто алгебраической природы.

\section{§3. Пример основной задачи}

3.1. Дилатация классической алгебры Гекке. Комплексная алгебра Гекке $H_{n}(q), q \in(0, \infty), n \geqslant 2, n \in \mathbb{N}$, задается образующими $\tau_{i}, i=1, \ldots, n-1$, и соотношениями

$$
\begin{gathered}
\tau_{i} \tau_{j}=\tau_{i} \tau_{j}, \quad|i-j|>1, i, j=1, \ldots, n-1, \\
\left(\tau_{i}+1\right)\left(\tau_{i}-q\right)=0, \quad i=1, \ldots, n-1, \\
\tau_{i} \tau_{i+1} \tau_{i}=\tau_{i+1} \tau_{i} \tau_{i+1},
\end{gathered}
$$

Хорошо известно, что эта алгебра изоморфна групповой алгебре $\mathbb{C}\left[\mathfrak{S}_{n}\right]$ симметрической группы $\mathfrak{S}_{n}$, а элементы $\tau_{i}, i=1, \ldots, n-1$, суть деформации классических коксетеровских транспозиций $\sigma_{i}$. Линейный базис алгебры Гекке индексируется элементами симметрической группы: $\mathfrak{S}_{n} \ni g \rightarrow \tau_{g}$, причем коксетеровской транспозиции $\sigma_{i}=(i, i+1) \in \mathfrak{S}_{n}$ сопоставляется образующая $\tau_{\sigma_{i}} \equiv \tau_{i}, i=1, \ldots, n-1$, а произвольной подстановке $g$ с приведенным разложением в произведение коксетеровских транспозиций $g=\prod_{k} \sigma_{i_{k}}$ сопоставляется элемент $\tau_{g}=\prod_{k} \tau_{i_{k}}$. Рассмотрим в этой алгебре покоординатное кокоммутативное коумножение $\Delta$, т. е. диагональ на указанном базисе $\left\{\tau_{g}\right\}$ :

$$
\Delta\left(\tau_{g}\right)=\tau_{g} \otimes \tau_{g}, \quad g \in \mathfrak{S}_{n} .
$$

Поскольку при $q \neq 1$ линейный базис $\left\{\tau_{g}\right\}, g \in \mathfrak{S}_{n}$, не образует группу (произведение двух его элементов $\tau_{h_{1}} \cdot \tau_{h_{2}}=\sum_{g} c_{h_{1}, h_{2}}^{g} \tau_{s}$ есть лишь линейная комбинация элементов базиса), это коумножение уже не является гомоморфизмом алгебры $H_{n}(q)$ в алгебру $H_{n}(q) \otimes H_{n}(q)$, и потому алгебра Гекке не является биалгеброй и тем более алгеброй Хопфа, каковыми являются групповые алгебры $\mathbb{C}\left[\mathfrak{S}_{n}\right]$. В то же время, если $q$ есть степень простого числа $p, q=p^{m}$, то классический результат состоит в том, что алгебра Гекке $H_{n}(q)$ есть подалгебра групповой биалгебры $\mathbb{C}\left[G L_{n}\left(F_{q}\right)\right]$ группы всех обратимых матриц $G L_{n}\left(F_{q}\right)$ над конечным полем $F_{q}$, а именно подалгебра двусторонних классов по борелевской подгруппе (группе верхнетреугольных матриц); при этом коумножение в $H_{n}(q)$, определенное выше, есть проекция коумножения в групповой алгебре. Таким образом, алгебра Гекке $H_{n}(q)$ при $q=p^{m}$ вкладывается в групповую алгебру группы $G L_{n}\left(F_{q}\right)$.

Однако при $q>1$ коумножение в алгебре Гекке, не являясь гомоморфизмом, все же остается положительным отображсением в смысле конуса положительных элементов (относительно естественной инволюции), поскольку, как 
легко видеть, коэффициенты $c_{g, h}^{s}$ - структурные константы умножения - неотрицательны. Очевидно, что положительность коумножения и умножения является необходимым условием вложения в биалгебру, так как гомоморфизмы всегда являются положительными отображениями и положительность сохраняется при положительных проекциях (но, конечно, не всякое положительное отображение мультипликативно). Пользуясь языком теории операторов, можно сказать, что при $q=p^{m}$ удалось найти дилатацию (подъем) алгебры Гекке: исходные положительные, но не мультипликативные операции - умножение и коумножение - в $H_{n}(q)$ стали в объемлющей групповой биалгебре $\mathbb{C}\left[G L_{n}\left(F_{q}\right)\right]$ ограничением и проекцией мультипликативных операций ${ }^{1)}$.

Поэтому возникает естественный вопрос: не является ли положительность умножения и коумножения достаточным условием вложимости в какую-либо биалгебру? Более конкретно:

нет ли подобного вложения и для других значений $q \neq p^{m}$, т.е. нельзя ли вложить алгебру Гекке $H_{n}(q)$ при других q с исходным умножением $и$ дилатацией коумножения в некоторую биалгебру, не обязательно групповую, или в алгебру Хопфа, бъть может, даже бесконечномерную?

Постановка вопроса требует уточнения того, что значит «вложение»или «дилатация»; для этого мы и определяем понятия подобъекта биалгебр. Предварительный анализ этих понятий и частичное решение вопроса для двумерных позитивных 2-алгебр - основной предмет этой статьи.

Существование строгого (см. далее) вложения алгебр Гекке в групповую алгебру конечной группь для общих $q$ маловероятно; например, для $n=3, q \neq p^{m}$ вложений в групповые биалгебры не существует (замечание И. Н. Пономаренко). Наоборот, скорее существование такой группы $\left(G L_{n}(q)\right)$ при $q=p^{m}$ выглядит удивительным. И хотя, как мы увидим, инволютивные полупростые биалгебры не исчерпываются групповыми биалгебрами и полное описание этого класса, данное здесь, показывает, что он совпадает с классом полугрупповых биалгебр конечных инверсных полугрупп, даже это не увеличивает возможностей для положительного решения вопроса.

Описание препятствий для таких вложений было бы не менее интересным для анализа таких объектов. Они встречаются уже в размерности 2 (см. разд. 6.4). Очевидным препятствием к построению конечномерных накрывающих алгебр служит иррациональность структурных констант коумножения; препятствием к построению групповых дилатаиий служит нарушение некоторых арифметических связей между структурными константами, или же такое препятствие может быть связано с условиями на порядки групп и подгрупп и

1) Приведем примеры употребления термина «дилатация». Унитарная дилатация сжатия $T$ в гильбертовом пространстве $K$ есть унитарный оператор $U$ в некотором расширении $H \supset K$, такой, что для ортогонального проектора $P: H \rightarrow K$ и всех натуральных $n$ имеет место равенство $T^{n}=P U^{n}$ (теоремы типа теорем Наймарка, Надя и др.). Более близкая к рассматриваемой ситуация: дилатацией положительного (марковского) оператора $T$ в пространстве $L^{2}$ называется унитарный мультипликативный оператор $U$ с аналогичным свойством: $T^{n}=P U^{n}$ для некоторого математического ожидания $P$ (см. [31]). В нашем случае речь идет о дилатации только коумножения как операции из алгебры в ее тензорный квадрат, так как умножение непосредственно наследуется из расширенной биалгебры. Дилатациям также посвящены работы М. Г. Крейна и Фояша-Надя, см. [18]. В отличие от известных теорем о дилатациях одного оператора, мы ищем, например, согласованные дилатации семейства операторов умножения на каждый из элементов базиса алгебры. 
т. д. Так или иначе, общий ответ о возможности вложений алгебр Гекке в биалгебры автору неизвестен.

3.2. Вероятностная трактовка: дерандомизация случайного умножения. На наш пример с алгеброй Гекке можно посмотреть иначе. Сделаем замену образующих:

$$
\bar{\tau}_{g}=q^{-l(g)} \tau_{g}, \quad g \in \mathfrak{S}_{n},
$$

где $l(g)$ - приведенная длина подстановки $g$, в частности, $\bar{\tau}_{i}=\frac{\tau_{i}}{q}, \bar{e}=e$. После этой нормировки в формуле

$$
\bar{\tau}_{h_{1}} \cdot \bar{\tau}_{h_{2}}=\sum_{g} \mu_{h_{1}, h_{2}}^{g} \bar{\tau}_{g}
$$

для умножения элементов нового базиса, как нетрудно проверить, сумма коэффициентов $\sum_{s} \mu_{h_{1}, h_{2}}^{g}$ (все они неотрицательны) стала равной единице; поэтому можно считать, что произведение любых двух элементов нового базиса есть вероятностная мера $\mu_{h_{1}, h_{2}}$ на множестве $\left\{\tau_{g}\right\}, g \in \mathfrak{S}_{n}$. Иначе говоря, умножение является «вероятностным», или «случайным», в противоположность умножению в группах или полугруппах, где оно является в понятном смысле «детерминированным».

Назовем устойчивым разбиением группы, или полугруппы, или более общей системы с бинарной операцией такое разбиение этой системы на подмножества (блоки), что векторное подпространство групповой алгебры, состоящее из всех линейных комбинаций блоков, есть алгебра. Тогда поставленный выше вопрос о дилатации умножения можно сформулировать так. Пусть задано «случайное» умножение на множестве $\mathfrak{S}_{n}$ (или на другом множестве); следовательно, векторное пространство функций на этом множестве есть алгебра. Не является ли умножение в этой алгебре проекцией (рандомизацией) детерминированного умножения? Более подробно, существуют ли такие группа или полугруппа (или даже $G$-пространство) и ее устойчивое разбиение, что для любых двух блоков разбиения $(s$ и $t$ ) распределение их произведения как меры на множестве блоков совпадает с правилом умножения в исходной алгебре (мерой $\mu_{s, t}$ в приведенном выше примере). В этой трактовке наш вопрос заключается в том, как дерандомизировать случайное умножение или коумножение. Аналогичные более простые задачи о дерандомизации встречаются, например, в общей теории марковских процессов, эргодической теории и др. Именно такая дерандомизация предлагается в конце статьи. Еще одна - комбинаторная - переформулировка нашей задачи состоит в следующем: возможно ли тензоры умножения и коумножения некоторой позитивной 2-алгебры (см. далее) с рациональными вещественными коэффициентами представить как факторы тензоров умножения и коумножения с коэффициентами 0, 1 ? Этот аспект проблемы будет рассмотрен отдельно.

\section{§4. Биалгебры, инволютивные биалгебры}

\section{1. Биалгебры.}

Определение 1. 2-алгеброй мы называем векторное пространство над полем $\mathbb{C}$, в котором заданы структуры ассоциативной алгебры с единицей и коассоциативной коалгебры с коединицей над тем же полем (вообще говоря, без каких бы то ни было условий согласованности этих структур между собой). 
В дальнейшем, если не оговорено противное, рассматриваются только конечномерные 2-алгебры, которые являются полупростыми алгебрами и коалгебрами (коалгебра полупроста, если полупроста двойственная ей алгебра). Биалгеброй (см. [8]) называется 2-алгебра, удовлетворяющая следующим эквивалентным условиям:

1) коумножение и коединица являются гомоморфизмами соответствующих алгебр;

2) умножение и единица являются гомоморфизмами соответствующих коалгебр ${ }^{1)}$.

Обозначим умножение как оператор из $A \otimes A$ в $A$ через $\delta$, единицу через $\epsilon$, коумножение через $\Delta: A \rightarrow A \otimes A$, а коединицу через $\varepsilon$. Определение биалгебры симметрично по отношению к парам $(\Delta, \varepsilon)$ и $(\delta, \epsilon)$, что часто делает излишними доказательства параллельных утверждений. Иногда произведение в $A$ мы будем записывать также обычным образом: $\delta(x \times y)=x \cdot y$.

Полезно связать все эти и последующие определения с определениями пар конечномерных алгебр в двойственности. Пусть $A$ есть 2-алгебра. Напомним, что в конечномерном случае двойственное пространство к алгебре (коалгебре) есть коалгебра (соответственно алгебра). Определим умножение в двойственном векторном пространстве $A^{\prime}$ с помощью отображения, сопряженного с коумножением $\Delta$, инволюцию - как операцию, сопряженную с коинволюцией в $A$, и единицу - с помощью коединицы в $A$. Тогда можно говорить о паре алгебр $A$ и $A^{\prime}$ в двойственности с теми или иными дополнительными свойствами (мультипликативность, положительность и т.д.). И наоборот, упорядоченная пара алгебр в невырожденной двойственности порождает 2-алгебру. Этот переход будет использоваться далее.

Групповая алгебра конечной группы со сверточным умножением и диагональным коумножением, очевидно, является кокоммутативной биалгеброй (и даже алгеброй Хопфа). Хорошо известно также (см. [8]), что полугрупповая алгебра любой конечной полугруппы (моноида) с единицей есть также кокоммутативная биалгебра с естественными определениями операций. В дальнейшем мы будем говорить о групповых или полугрупповых биалгебрах, имея в виду, что на них рассматриваются обе мультипликативные структуры.

4.2. Инволютивные биалгебры. Наш план состоит во введении дополнительной структуры в биалгебрах, так или иначе присутствующей в групповых и близких к ним биалгебрах, и в последующем ослаблении некоторых требований к структурам биалгебр. Снабдим биалгебру $A$ инволюцией и коинволюцией; инволюцией алгебры $A$ называется антилинейный антиавтоморфизм второго порядка этой алгебры; точно так же антилинейный антиавтоморфизм второго порядка коалгебры $A$ называется коинволюцией. Алгебры Хопфа с инволюцией (но без коинволюции) рассматривались ранее, см., например, [8]. Будем обозначать инволюцию и коинволюцию в $A$ через $\sharp$ и b соответственно. В этих обозначениях связи инволюции и коинволюции с умножением и коумножением выглядят так:

$$
\delta(\sharp \otimes \sharp)=\sharp \delta J, \quad(b \otimes b) \Delta=J \Delta b,
$$

1) Естественно было бы использовать термин «биалгебра» для объектов, названных выше 2-алгебрами, а ту или иную согласованность умножения и коумножения отмечать эпитетом к слову «биалгебра»; тогда то, что называется сейчас биалгеброй, следовало бы назвать «мультипликативной биалгеброй», то, что далее названо «позитивной 2-алгеброй», - «позитивной биалгеброй» и т. д. Но такая переделка терминологии, к сожалению, слишком запоздала. 
где $J$ - перестановка (флип) в тензорном произведении: $J(x \otimes y)=y \otimes x$.

Определение 2. Биалгебра, снабженная инволюцией и коинволюцией, называется инволютивной биалгеброй или биалгеброй с инволюиией, если умножение коммутирует с коинволюцией, а коумножение - с инволюцией:

$$
\Delta \sharp=(\sharp \otimes \sharp) \Delta, \quad \delta(b \otimes b)=b \delta .
$$

В алгебре с инволюцией $\sharp$ определено понятие неотрицательных элементов - элементов вида $x \cdot x^{\sharp}$. Они образуют выпуклый конус $K^{\sharp}$, который мы будем называть положительным конусом относительно инволюиии $\sharp$ (в конечномерном случае он будет замкнутым). Положительный конус $K^{b}$ относительно коинволюиии b определяется следующим образом: это полный прообраз относительно коумножения $\Delta$ конической оболочки элементов вида $x \otimes x^{b} \in A \otimes A$, $x \in A$; иначе говоря, $K^{b}=\left\{y \in A: \Delta y=\sum_{i} a_{i} \otimes a_{i}^{b}\right\}^{1)}$.

Теорема 1. Коумножение в инволютивной биалгебре сохраняет конус, положительный относительно инволюиии, а умножение сохраняет конус, положительный относительно коинволюиии, или, коротко, в инволютивной биалгебре умножение и коумножение положительны.

Замечание. Это утверждение содержит, в частности, алгебраическую часть теоремы Крейна о положительности умножения и коумножения для конечномерного случая.

Доказательство. Нужно доказать, что образ относительно копроизведения элемента, неотрицательного относительно инволюции, лежит в положительном конусе и что произведение элементов, неотрицательных относительно коинволюции, лежит в соответствующем конусе. В силу симметрии достаточно доказать одно из утверждений, второе будет получаться из первого заменой коумножения на умножение и инволюции на коинволюцию и переходом к двойственной биалгебре. При доказательстве положительности копроизведения положительных элементов можно ограничиться элементами вида $x \cdot x^{\sharp}, x \in A$, так как они порождают конус положительных элементов. Следующая выкладка использует коммутационные соотношения, сформулированные выше:

$$
\Delta\left(x \cdot x^{\sharp}\right)=\Delta(x) \Delta\left(x^{\sharp}\right)=\Delta(x)[\Delta(x)]^{\sharp} .
$$

Мы использовали мультипликативность коумножения и его коммутирование с инволюцией; аналогично при доказательстве второго утверждения используется мультипликативность умножения (относительно коумножения) и его коммутирование с коинволюцией.

4.3. Инволютивные биалгебры и полугрупповые алгебры инверсных полугруппы. Комплексные групповые алгебры конечных групп с естественными структурами являются инволютивными биалгебрами в определенном выше смысле - инволюция порождена переходом к обратному в группе $g \rightarrow g^{-1}$, а коинволюция - сопряжением коэффициентов. Более того, инволютивная кокоммутативная полупростая конечномерная алгебра Хопфа является групповой биалгеброй конечной группы. Ниже мы рассмотрим класс конечномерных инволютивных полупростых кокоммутативных биалгебр и свяжем его с важным классом полугрупп.

1) В терминах двойственности этот конус есть просто дуальный конус к конусу положительных элементов в двойственной алгебре относительно инволюции, сопряженной с инволюцией b. 
А именно, мы покажем, что класс конечных инверсных полугрупп с единицей порождает в точности класс инволютивных полупростых биалгебр. Напомним (см. [11]), что инверсной полугруппой называется полугруппа $S$, в которой каждый элемент $a \in S$ имеет единственный инверсный элемент $b \in S$, $a b a=a$. Единственность этого элемента гарантирует, что инверсный к элементу $b$ элемент есть $a$; поэтому на инверсной полугруппе существует естественная инволюция: каждому элементу а сопоставляется инверсный ему, обозначаемый через $a^{*}=a^{-1}$. Умножение, коумножение и коинволюция определяются так же, как и в групповом случае. Если в полугруппе $S$ есть двусторонняя единица, то функционал «значение в единице» является коединицей (в коалгебре).

Основным и наиболее интересным для нас примером инверсной полугруппы служит симметрическая инверсная полугруппа $\mathscr{I}_{n}$ взаимно однозначных частичных отображений $n$-элементного множества в себя, т. е. биекций между его подмножествами с пустым отображением в качестве нуля полугруппы. Важная теорема, обобщающая теорему Кэли о группах, утверждает, что всякая конечная инверсная полугруппа изоморфно вкладывается в симметрическую инверсную полугруппу.

Пример. Рассмотрим инверсную полугруппу $\mathscr{I}_{n}^{1}$, состоящую из $n^{2}$ матричных единиц $\left\{e_{i, j}\right\}, i, j=1, \ldots, n$, и нуля 0 с обычным матричным умножением. Обратный элемент определяется так: $e_{i, j}^{*}=e_{j, i}, 0^{*}=0$. Полугрупповая алгебра $\mathbb{C}\left[\mathscr{I}_{n}^{1}\right]$ есть прямая сумма $M_{n}(\mathbb{C}) \oplus \mathbb{C}$ алгебры матриц и одномерного двустороннего идеала с кронекеровским (т. е. покоординатным в двойственной формулировке) коумножением. Сжатая полугрупповая биалгебра (т. е. фактор по одномерному идеалу, порожденному нулем полугруппы) является просто матричной биалгеброй $M_{n}(\mathbb{C})$ (с кронекеровским коумножением). Обе алгебры, как отмечалось выше, суть неунитальные инволютивные биалгебры. Полугруппа $\mathscr{I}_{n}^{1}$ есть подполугруппа упомянутой симметрической инверсной полугруппы $\mathscr{I}_{n}$.

Теорема 2. Полугрупповая биалгебра конечной инверсной полугруппь $S c$ единицей со сверткой в качестве умножения и поточечным коумножением есть полупростая кокоммутативная инволютивная биалгебра. Двойственная полугрупповая биалгебра $\mathbb{C}(S)$ есть полупростая коммутативная инволютивная биалгебра. Обратно, всякая конечномерная полупростая кокоммутативная (в двойственном варианте коммутативная) инволютивная биалгебра изоморфна (как инволютивная биалгебра) полугрупповой алгебре (соответственно двойственной полугрупповой алгебре) конечной инверсной полугруппъ с единиией.

Эту формулировку можно обобщить на инверсные полугруппы без единицы, надо лишь добавить, что полугрупповая алгебра не является в точном смысле слова биалгеброй, поскольку в ней или нет коединицы, или она не определяет гомоморфизм в поле.

Доказательство. Пусть $\mathbb{C}[S]$ есть полугрупповая биалгебра конечной инверсной полугруппы $S$ над полем $\mathbb{C}$. Теорема Оганесяна [17] утверждает, что полугрупповая алгебра всякой конечной инверсной полугруппы полупроста. Поэтому в проверке нуждается лишь то, что связано с инволюцией, а именно, что $(a b)^{*}=b^{*} a^{*}$, а это действительно верно (см. [11, лемма 1.18]); остальные свойства инволюции и коинволюции проверяются очевидным образом. Если в 
полугруппе $S$ нет двусторонней единицы, то нет и соответствующего гомоморфизма в $\mathbb{C}$, и в этом случае $\mathbb{C}[S]$, вообще говоря, ослабленная инволютивная биалгебра.

Для доказательства обратного утверждения нам удобнее рассматривать двойственную формулировку и двойственную алгебру $A^{\prime}$. Поскольку $A^{\prime}$ коммутативна и полупроста, то как алгебра с инволюцией она изоморфна алгебpe $\mathbb{C}^{n}$. В силу мультипликативности коумножения $\Delta$ образ идемпотента в $A$ относительно $\Delta$ есть идемпотент в $A \otimes A$, и поэтому коумножение определяет бинарную операцию на спектре алгебры $\mathbb{C}^{n}$, т. е. на $1, \ldots, n$. В силу коассоциативности коумножения $\Delta$ эта операция задает на спектре структуру конечной ассоциативной полугруппы, возможно, без единицы и, возможно, с нулем. Поскольку инволюция коммутирует с коумножением, то она определяет инволюцию на полугруппе. Тот факт, что эта полугруппа является инверсной, вытекает из того, что всякая конечная подполугруппа комплексных матриц, замкнутая относительно обычной инволюции матриц, является инверсной. Для того чтобы убедиться в этом, можно использовать следующий критерий инверсности: полугруппа с инволюцией является инверсной в том и только в том случае, если ее подполугруппа идемпотентов коммутативна (см. [11, теорема 1.17] или упомянутый аналог теоремы Кэли; см. также [20]). Наличие коединицы в биалгебре эквивалентно наличию единицы в полугруппе.

Мы охарактеризовали класс полупростых кокоммутативных конечномерных инволютивных биалгебр. В отличие от групповых биалгебр полугрупповые биалгебры инверсных полугрупп, вообще говоря, не являются алгебрами Хопфа, так как в них нет антипода. Но в них есть «почти антипод»: рассмотрим в полугрупповой алгебре инверсной полугруппы линейное продолжение $S$ операции перехода к инверсному элементу $a \rightarrow a^{-1}$. Этот оператор удовлетворяет условию, несколько отличающемуся от обычного условия на антипод (см. [8]), а именно, его свертки слева и справа с тождественным отображением, $S \star$ id и id $\star S$, суть проекции не на одномерное подпространство скаляров биалгебры, как это должно быть для антипода в алгебрах Хопфа, а на коммутативную подалгебру идемпотентов исходной полугруппы. Таким образом, в категории конечномерных инволютивных биалгебр класс полугрупповых биалгебр конечных инверсных полугрупп наиболее близок к классу алгебр Хопфа ${ }^{1)}$.

\section{§5. Позитивные 2-алгебры}

5.1. Определения. Теорема 1 дает основания для следующего основного определения:

Определение 3. Полупростая 2-алгебра $A$ над $\mathbb{C}$ с умножением $\delta: A \otimes A \rightarrow$ $A$, единицей $\epsilon$, инволюцией $\sharp$, коумножением $\Delta: A \rightarrow A \otimes A$, коединицей $\varepsilon$ и коинволюцией $b$ называется позитивной 2-алгеброй, если операции удовлетворяют таким условиям:

(1) умножение и коумножение положительны, т.е. умножение $\delta$ сохраняет конус $K^{b}, \delta\left(K^{b} \otimes K^{b}\right) \subset K^{b}$, а коумножение $\Delta-$ конус $K^{\sharp}, \Delta K^{\sharp} \subset K^{\sharp} \otimes K^{\sharp}$;

1) Заметим, что и теория представлений инверсных полугрупп частично изометрическими операторами очень близка к теории унитарных представлений групп; кроме того, полугрупповая биалгебра конечной инверсной полугруппы с единицей является планшерелевой в смысле [9], [6]. Все это, в частности, позволяет существенно упростить доказательства многих фактов, например, теоремы о полупростоте и других упомянутых выше свойств инверсных полугрупп. 
(2) как и в определении инволютивных биалгебр, инволюция $\sharp$ коммутирует с коумножением, $\Delta\left(x^{\sharp}\right)=\{\Delta(x)\}^{\sharp \otimes \sharp}$, а коинволюция $b-$ с умножением, $x^{b} \cdot y^{b}=$ $(x \cdot y)^{b}$.

Понятие позитивной 2-алгебры расширяет понятие инволютивной биалгебры, заменяя условия мультипликативности умножения и коумножения на более слабое условие положительности и той, и другой операции. В терминах двойственности алгебр оно было введено в [5]. Из теоремы 1 вытекает такое следствие:

Следствие 1. Инволютивная биалгебра удовлетворяет условиям (1), (2) u, тем самым, является позитивной 2-алгеброй.

Действительно, условие (1) следует из мультипликативности умножения и коумножения для биалгебр, а условие (2) входит в определение инволютивных биалгебр.

Условие (1) для позитивных 2-алгебр нельзя заменить условием положительности только одной операции: здесь уже из положительности одной операции положительность второй, вообще говоря, не следует, как показывают простые примеры (см., в частности, [3] и [6]), и это отличает позитивные 2-алгебры от биалгебр. Распознавание того, будет ли позитивной конечномерная 2-алгебра, по структурным тензорам, задающим умножение и коумножение, есть $N P$-полная задача (в отличие от проверки свойства 2-алгебры быть биалгеброй).

Если надо подчеркнуть наличие всех перечисленных структур в позитивной биалгебре $A$, мы будем использовать запись $A=A(\delta, \epsilon, \sharp, \Delta, \varepsilon, b)$.

Наиболее важен чуть более узкий класс инволютивных биалгебр и позитивных 2-алгебр, который получается добавлением еще двух двойственных между собой требований, связывающих обе структуры:

Определение 4. Позитивная 2-алгебра называется однородной, если кроме условий (1), (2) выполняется условие

(3) коединица есть положительный гомоморфизм алгебры в поле, $\varepsilon(x \cdot y)=$ $\varepsilon(x) \varepsilon(y)$, а образ единицы при коумножении есть единица в тензорном произведении, $\delta(\epsilon)=\epsilon \otimes \epsilon$. (Эти два условия симметричны относительно перехода к двойственным алгебрам и автоматически выполнены для биалгебр.)

Условие однородности (названное так позже в [6]) и было первоначальным условием положительной двойственности для пар алгебр в исходной работе [5]. Оно означает, что не только конус положительных (коположительных) элементов замкнут относительно коумножения (умножения), но замкнуты относительно этих операций также компакты состояний (т. е. нормированных положительных функционалов). Это позволяет развить так называемую геометрическую теорию состояний или теорию пакетов (см. далее и [5], [9], [10]). В дальнейшем, если не оговорено противное, мы рассматриваем только однородные позитивные 2-алгебры ${ }^{1)}$. В недавней работе [21] (см. список литературы в ней) были описаны групповые алгебры $n$-значных групп ( $n$-алгебры Хопфа). Это описание дается в терминах гомоморфизмов Фробениуса. Поскольку групповые

1) Не останавливаясь на деталях, отметим, что, аналогично доказанному в [6] факту, категория однородных конечномерных полупростых позитивных 2-алгебр с дополнительным условием планшерелевости (которое является условием общего положения) эквивалентна категории конечномерных алгебр в планшерелевой двойственности в смысле [5], [10] и [6] и категории положительных $C$-алгебр [2]. 
алгебры инволютивных $n$-значных групп являются частным случаем позитивных 2-алгебр (это случай, когда структурные константы рациональны), было бы интересно распространить это описание на все позитивные 2-алгебры.

Несколько вольно различие между биалгебрами и однородными позитивными 2-алгебрами можно выразить следующим образом: умножение (и коумножение) в биалгебрах является «детерминированным», а в позитивных 2-алгебрах - «вероятностным» (многозначным) (см. §3).

5.2. Алгебры в положительной двойственности ([5], [15]). Понятие позитивной 2-алгебры есть калька понятия пары алгебр в положительной двойственности, введенного в [5]. Совсем кратко приведем их определение. Пусть $A$ и $B$ - две инволютивные алгебры над $\mathbb{C}$ с единицами и определено их невырожденное комплексное спаривание $\langle A, B\rangle$. Эта пара алгебр находится в однородной положительной двойственности, если выпуклое подмножество в $B$ состояний (= нормированных положительных функционалов) на алгебре $A$ устойчиво относительно инволюции и умножения в $B$, выпуклое подмножество в $A$ состояний на алгебре $B$ устойчиво относительно инволюции и умножения в $A$ и единица алгебры $A$ (соответственно алгебры $B$ ) есть коединица алгебры $B$ (соответственно алгебры $A$ ). Положительная двойственность называется неоднородной или ослабленной, если устойчивы (в том же смысле) только конусы неотрицательных элементов и ничего не предполагается о единицах. Если заменить операцию умножения в $B$ (соответственно в $A$ ) сопряженной с ней операцией коумножения в $A$ (соответственно в $B$ ), то получившаяся в $B$ (соответственно в $A$ ) структура 2 -алгебры превратит $B$ (соответственно $A$ ) в позитивную 2-алгебру в смысле определения, данного в разд. 5.1.

Язык двойственности подчас удобнее языка биалгебр. Например, он позволяет развить содержательное исследование конечномерной и бесконечномерной геометрии пар пакетов, которая рассматривалась в [5] как геометрическая теория состояний на *-алгебрах. Пакет - это выпуклый компакт определенного вида (блок-симплекс) состояний на полупростой конечномерной $*$-алгебре, снабженный умножением. Геометрия пакетов нетривиальна уже в коммутативном случае, пакет в этом случае есть аффинный симплекс со структурой полугруппы с инволюцией. Эта геометрия была далеко продвинута в [9], [10]. Соответствующие определения охватывают как частный случай групповые алгебры, алгебры, возникающие в алгебраической комбинаторике (клеточные алгебры) и некоторые новые примеры. Можно ввести дальнейшие ограничения на связь умножения и коумножения (планшерелевость и др.); они ранее по инициативе автора были подробно рассмотрены в работах [9], [10] и модифицированы в недавних работах, связанных с алгебраической комбинаторикой, многозначными группами и др., см. [3], [6], [7] и [21]; мы не рассматриваем здесь этих вопросов.

\section{§6. Подобъекты инволютивных биалгебр и формулировка основной проблемы}

6.1. Строгие подобъекты. Перед тем как сформулировать центральную проблему, мы должны определить понятие подобъекта в категории инволютивных биалгебр. Автору неизвестно, определялось ли такое понятие в категории биалгебр или алгебр Хопфа. Возможны различные понятия подобъекта; мы рассмотрим два из них. Пусть $A-$ инволютивная биалгебра, и предположим, что 
$B$ есть подалгебра с единицей биалгебры $A$ (как алгебры), замкнутая относительно инволюции и коинволюции. Если выполнено условие

$$
\Delta B \subset B \otimes B,
$$

то коумножение в $B$ наследуется из $A$ и поэтому автоматически выполнены условия коассоциативности и коммутирования с инволюцией. В этом случае $B$ есть инволютивная биалгебра со структурами, наследуемыми из биалгебры $A$, и, казалось бы, естественно считать ее подобъектом в $A$. Однако это условие почти никогда не выполняется, класс таких подобъектов слишком узок и не покрывает наиболее важных и нетривиальных приложений. Предположим, что вместо условия (*) выполнено гораздо более слабое условие:

Определение 5. Подалгебра $B$ с единицей биалгебры $A$, замкнутая относительно инволюции и коинволюции, называется строгим подобъектом биалгебры $A$ или допустимой подалгеброй инволютивной биалгебры $A$, если существует коидеал $J$ в $A$, также замкнутый относительно инволюции и коинволюции, такой, что $A$ разлагается в прямую сумму подалгебры $B$ и коидеала $J$. Двойственное определение: строгий подкообъект биалгебры - это ее подкоалгебра, для которой существует такой идеал, что биалгебра разлагается в прямую сумму этих подкоалгебры и идеала.

При естественном отождествлении факторкоалгебры $A / J$ коалгебры $A$ по коидеалу $J$ с подалгеброй $B$ последняя получает структуру коалгебры и превращается в позитивную 2-алгебру (см. ниже); вообще говоря, она не является биалгеброй. Это определение можно высказать иначе. Пусть $B$ есть подалгебра биалгебры $A$ (как алгебры), содержащая единицу и замкнутая относительно инволюции и коинволюции. Предположим, что в $A$ существует положительный оператор $P=P_{B}$ проектирования на подалгебру ${ }^{1)} B$, такой, что отображение $\Delta_{P_{B}}: B \rightarrow(P \otimes P) \Delta$, т. е. $P$-проекция коумножения $\Delta$ на подалгебру $B$, задает коассоциативное коумножение на $B$, и предположим, что ограничение коединицы в $A$ на $B$ есть коединица относительно этого коумножения.

Предложение 1. Умножение $\left.\delta\right|_{B}$ и коумножение $\Delta_{P}$ на допустимой подалгебре $B$, введенные выше, положстельны относительно коинволюиии и инволюиии соответственно.

Доказательство. В проверке нуждается лишь положительность коумножения, но положительная проекция (= математическое ожидание) переводит положительные операции (каковой является мультипликативное коумножение) в положительные.

Отсюда и из непосредственной проверки условия (3) вытекает

Следствие 2. Допустимая подалгебра инволютивной биалгебры является однородной позитивной 2-алгеброй.

Замечание. 1) Условие допустимости подалгебры $B \subset A$ очень ограничительно: хотя для всякой подалгебры существует, как правило, единственный положительный проектор $P_{B}$, коассоциативность проекции коумножения $\left(P_{B} \otimes P_{B}\right) \Delta$ имеет место лишь в частных случаях (см. следующий раздел).

1) $P_{B}$ есть положительный оператор математического ожидания. 
2) Кажущаяся асимметрия между умножением и коумножением в нашем определении вызвана только удобством формулировок - легко сформулировать эквивалентное двойственное определение, приводящее к тому же классу подобъектов.

Сформулируем предыдущее определение в терминах пар алгебр в положительной двойственности с тем, чтобы затем в тех же терминах дать более широкое определение подобъекта.

Рассмотрим инволютивную биалгебру $\widehat{A}$ над $\mathbb{C}$ как пару алгебр в двойственности $\left\langle A, A^{\prime}\right\rangle$, где $A$ рассматривается как $\widehat{A}$ со структурой алгебры, а $A^{\prime}$, (алгебраически) двойственное векторное пространство, снабжено структурой алгебры с умножением, двойственным к коумножению в $\widehat{A}$. Пусть $\widehat{B}-$ позитивная 2-алгебра, и пусть ей в том же смысле соответствует пара алгебр $\left\langle B, B^{\prime}\right\rangle$ в двойственности. По сказанному выше в обоих случаях (во втором из них по определению) двойственность алгебр положительная. Следующее утверждение есть тавтологическая переформулировка приведенного выше определения подобъекта.

Предложение 2. Пусть $T$ - изоморфное положительное вложение алгебры $B$ в алгебру $A$, сохраняющее единичу и такое, что ограничение сопряженного отображсения $T^{\prime}: A^{\prime} \rightarrow B^{\prime}$ на некоторую подалгебру $C$ алгебры $A^{\prime}$ есть изоморбизм этой подалгебры на алгебру $B^{\prime}$ и $T^{\prime}$ является оператором математического ожидания на $C \subset A^{\prime}$. Тогда

1) при отождествлении подалгебры $C$ и алгебры $B^{\prime}$ с помощъю $T^{\prime}$ умножение в $B^{\prime}=C$ соответствует коумножению в $B$.

2) $T \widehat{B}$ есть подобгект в $\widehat{A}$.

Обратно, для всякого подобгекта $\widehat{B}$ биалгебры $\widehat{A}$ оператор $T$ вложения $B$ как алгебры в $A$ обладает указанным свойством.

6.2. Динамические вложения в инволютивные биалгебры: нестрогие подобъекты. Оказывается, что для решения проблемы дилатации предыдущее определение подобъекта также слишком жесткое. Как мы увидим, положительное решение возможно крайне редко даже в малых размерностях, а условия существования такого решения, по-видимому, трудно выразимы. Дадим более широкое понятие подобъекта, которое можно было бы назвать динамическим. Точнее, определим новое понятие вложения позитивных 2-алгебр в инволютивные биалгебры. По поводу понятий комодуля и кодействия см. [8]; эти определения легко интерпретировать в терминах пар алгебр в двойственности.

Определение 6. Скажем, что позитивная 2-алгебра $\widehat{B}$ нестрого вкладывается в биалгебру $\widehat{A}$ или что ее образ есть нестрогий подобъект биалгебры $\widehat{A}$, если существует такое положительное сохраняющее единицу, инволюцию и коинволюцию изоморфное вложение алгебр $T: \widehat{B} \rightarrow \widehat{A}$, что (левое) кодействие $\widehat{B}$ как коалгебры на себе расширяется с алгебры $T \widehat{B}$ до (левого) кодействия всей коалгебры $\widehat{A}$ на себе.

В определении строгого подобъекта коумножение в подалгебре поднималось (с помощью проектора на подалгебру) до коумножения во всей биалгебре; в данном определении поднимается лишь кодействие; при этом способ поднятия никак не оговаривается. Можно сказать, что нестрогое вложение 2-алгебр есть мономорфизм структур алгебр и структур комодулей. Различие между двумя 
понятиями вложения видно уже на примерах бикоммутативных 2-алгебр, где умножение и коумножения коммутативны (см. разд. 6.4). Двойственная формулировка получается, если перейти к двойственным коалгебрам.

6.3. Формулировка основной проблемы. Теперь мы готовы точно сформулировать проблему вложения, или дилатации, позитивных 2-алгебр в биалгебры.

6.3.1. Строгий вариант. Пусть $A=A(\delta, \epsilon, \sharp, \Delta, \varepsilon, b)-$ конечномерная однородная позитивная 2-алгебра. Когда существует (возможно, ослабленная) инволютивная биалгебра $\mathscr{A}$, такая, что А изоморфна некоторому строгому под-

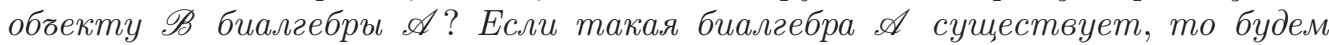
говорить, что есть строгая дилатация позитивной 2-алгебръ А. Когда существует минимальная каноническая строгая дилатация?

Будем называть как эту, так и следующую проблемы проблемой подъема позитивной 2-алгебры в биалгебру или проблемой строгой дилатаиии позитивной 2-алгебры, а в вероятностных терминах проблемой дерандомизаиии вероятностного коумножения. Свойство однородности позволяет интерпретировать проблему дилатации как проблему «дерандомизации» вероятностного коумножения (см. §3).

Если искать решение проблемы в классе конечномерных инволютивных биалгебр, то заведомо необходимо ввести дополнительные ограничения на позитивную 2-алгебру - у нее должны быть образующие с рациональными структурными константами; иррациональность констант при любых образующих требует перехода к бесконечномерным алгебрам.

С учетом теоремы 2 основная проблема, сформулированная выше, может быть поставлена гораздо более конкретно: какие конечномерные полупростые кокоммутативные однородные позитивные 2-алгебры с рациональными структурными константами изоморфны допустимой подалгебре двойственной полугрупповой биалгебры инверсной полугруппы (в частности, группъ)? Иначе говоря, когда существует строгая дилатаиия в полугрупповую биалгебру инверсной полугруппы какой-либо рациональной коммутативной позитивной 2-алгебры? По аналогии с группами про соответствующие позитивные 2-алгебры можно было бы сказать, что они имеют полугрупповое происхождение. Примеры ниже показывают, что такое расширение класса алгебр недостаточно для положительного решения проблемы, а для двумерных алгебр это расширение не дает ничего нового по сравнению с групповыми биалгебрами - см. п. 6.4.2.

Не представляет труда сформулировать проблему в терминах тензоров структурных констант операции коумножения в базисе минимальных идемпотентов по умножению и придать затем самой проблеме вид задачи о разложении тензоров коумножения позитивной 2-алгебры по тензорам Кэли групповой операции. Такая формулировка лишь подчеркивает сложность задачи, но вряд ли дает способ ее решения.

6.3.2. Нестрогий вариант. Изменим формулировки задач в предыдущем пункте, заменив везде слова «строгий подобъект», «строгая дилатация» и т. д. на слова «нестрогий подобъект», «нестрогая дилатация» и т. д., и мы получим нестрогий вариант проблемы. Различие лишь в том, что понятие подобъекта здесь более широкое. Это - менее жесткая постановка вопроса, такую дилатацию назовем еще динамической; это понятие ближе к понятию дилатации в 
смысле теории операторов [18]. Однако запас позитивных 2-алгебр, для которых нестрогая дилатация возможна, гораздо шире, чем в первом случае; это видно уже при рассмотрении бикоммутативных позитивных 2-алгебр - см. следующий раздел. Нестрогий вариант в его геометрической формулировке также иллюстрируется примером из п. 6.4.2.

\section{4. Примеры строгих и нестрогих подобъектов.}

6.4.1. Позитивные 2-алгебры, являющиеся строгими подобъектами инволютивных биалгебр. Комплексная групповая алгебра $\mathbb{C}[G]$ конечной группы $G$, рассматриваемая как алгебра формальных сумм элементов группы (или как алгебра комплексных мер на группе $G$ с коумножением, которое переносит такую меру с группы $G$ на диагональ прямого произведения $G \times G$, и обычными инволюцией и коинволюцией), есть инволютивная биалгебра (и даже алгебра Хопфа с антиподом $g \rightarrow g^{-1}$ ). Образ элемента $g$ группы при коумножении есть сумма пар элементов этой группы, произведение которых равно $g$.

Двойственная инволютивная биалгебра к групповой алгебре конечной группы есть коммутативная инволютивная биалгебра: это пространство всех комплексных функций на группе с поточечным умножением и диагональным коумножением $\Delta: \mathbb{C}(G) \rightarrow \mathbb{C}(G) \otimes \mathbb{C}(G),(\Delta f)(g, h)=f(g h)$, с комплексным сопряжением функций в качестве инволюции и с заменой аргумента функции на обратный с комплексным сопряжением в качестве коинволюции. Единица есть функция, тождественно равная единице, а коединица - функционал«значение функции в единице группы».

Общий пример допустимой подалгебры (строгого подобъекта) групповой биалгебры таков.

Предложение 3. Подалгебра $\mathbb{C}[H \backslash G / H] \equiv \mathscr{B}_{H}$ двусторонних классов по подгруппе $H$ (иногда называемая алгеброй Гекке, отвечающей подгруппе $H$ ) в групповой алгебре $\mathbb{C}[G]$ есть позитивная 2-алгебра, являющаяся допустимой подалгеброй биалгебры $\mathbb{C}[G]$ в смысле, определенном выше.

Доказательство. Проекция $P$ в $\mathbb{C}[G]$ на $\mathscr{B}_{H}$ есть усреднение по двойным классам по подгруппе; очевидно, что эта проекция является положительным математическим ожиданием, т. е. $P(x a y)=x P(a) y$ для всех $\left.x, y \in \mathscr{B}_{H}, a \in \mathbb{C}[G]\right)$, которое проектирует коумножение в алгебре $\mathbb{C}[G]$ в естественное коумножение на пространстве двойных классов (двойственное к умножению классов). Замкнутость относительно инволюций и коинволюций и наличие единиц и коединиц очевидны, как и выполнение соотношений между ними.

Предыдущее предложение остается дословно верным, если заменить разбиение на двойные классы смежности по подгруппе произвольным устойчивым разбиением группы, т. е. таким разбиением, что пространство функций, постоянных на блоках разбиения, является инвариантной подалгеброй групповой алгебры, содержащей единицу; и тогда это пространство также есть позитивная 2-алгебра относительно индуцированных структур. Примером устойчивых разбиений, отличных от разбиения на двойные классы по некоторой подгруппе, может служить разбиение на орбиты некоторой группы автоморфизмов группы.

Можно распространить этот пример на инверсные полугруппы. По теореме 2 полугрупповые биалгебры этих полугрупп являются инволютивными и полупростыми, и в них можно рассматривать подобъекты в предыдущем понимании. Легко проверить, что устойчивому разбиению инверсной полугруппы, понимаемому в том же смысле, что и в случае групп, соответствует допустимая 
подалгебра (подобъект) полугрупповой биалгебры, структурные константы такой 2-алгебры рациональны. Доказательство теоремы 2 фактически содержит утверждение, что допустимой подалгебре двойственной полугрупповой алгебры отвечает устойчивое разбиение инверсной полугруппы. Таким образом, получаем

Следствие 3. Класс строгих подобвектов инволютивных конечномерных полупростых биалгебр совпадает с классом позитивных 2-алгебр, порождаемых устойчивыми разбиениями конечных инверсных полугрупп (в частности, zpynn).

Действительно, по теореме 2 по такой биалгебре сначала строится инверсная полугруппа, а затем в ней - устойчивое разбиение. В бикоммутативном случае запасы строгих подобъектов для групповых и полугрупповых биалгебр совпадают. Поэтому в этом случае основная задача (о дилатации или дерандомизации коумножения) свелась к описанию примеров группового происхождения (шуровских алгебр). Именно эти примеры усиленно изучались в работах по теории ассоциативных схем, но в другой интерпретации и с другой терминологией (см. [6]). Однако это лишь небольшая часть позитивных 2-алгебр (или, на другом языке, клеточных алгебр, $C$-алгебр и т. д.). Подчеркнем, что теория ассоциативных схем занимается не просто алгебрами того или иного класса, но их целочисленными представлениями. Рассмотрения этой работы носят алгебраический характер; вопрос о представлениях алгебр, тем более целочисленных, следует рассматривать особо. Отметим, что проблема о строгих дилатациях может быть поставлена в тензорных терминах (в терминах структурных констант умножения и коумножения) аналогично тому, как ниже переформулирована в терминах матрицы квазихарактеров задача о нестрогой дилатации для бикоммутативных 2-алгебр; этот вопрос будет рассмотрен особо.

6.4.2. Классификация двумерных алгебр Гекке как инволютивных позитивных 2-алгебр. В этом пункте мы классифицируем двумерные позитивные 2-алгебры. Мы увидим на их примере, что класс нестрогих подобъектов биалгебр отличается от класса строгих подобъектов. Всякая двумерная позитивная 2-алгебра, очевидно, бикоммутативна и с точностью до изоморфизма описывается следующим образом. Как векторное пространство это комплексное двумерное пространство $\mathbb{C}^{2}$. Структуру алгебры зададим образующими - единицей алгебры 1 и элементом u с соотношением

$$
\mathbf{u}^{2}=(1-\lambda) \cdot \mathbf{u}+\lambda \cdot \mathbf{1}, \quad \lambda \in[0,1] .
$$

Структура коалгебры задается формулами

$$
\Delta(\mathbf{1})=\mathbf{1} \otimes \mathbf{1}, \quad \Delta(\mathbf{u})=\mathbf{u} \otimes \mathbf{u} .
$$

Инволюция и коинволюция суть комплексное сопряжение, коединица $\varepsilon$ задана равенствами $^{1)} \varepsilon(\mathbf{u})=-\lambda, \varepsilon(\mathbf{1})=1$. Обозначим полученную 2 -алгебру через $A_{\lambda}$. Легко проверить, что $A_{\lambda}$ - позитивная 2-алгебра, так как выполнены все аксиомы разд. 5.1. Заметим, что умножение задает алгебру усеченных полиномов второй степени от переменной $u$. В стандартном базисе пространства $\mathbb{C}^{2}$, которое понимается как коалгебра, единица имеет вид $\mathbf{1}=(1,1)$, а образующая $u$ имеет координаты $(-\lambda, 1)$. Легко доказать следующее утверждение:

1) В двойственном описании образующие идемпотенты алгебры суть $\varepsilon$ и $v$, а коумножение и единица задаются формулами $\Delta \varepsilon=\varepsilon \otimes \varepsilon, \Delta(v)=v \otimes 1+1 \otimes v+(1-\lambda) v \otimes v, \mathbf{1}(\varepsilon)=1$, $\mathbf{1}(v)=0$. 
Предложение 4. Всякая двумерная позитивная полупростая 2-алгебра изоморфна одной из 2-алгебр $A_{\lambda}, \lambda \in[0,1]$. При $\lambda=1$ это групповая алгебра группы второго порядка, а при $\lambda=0$ - полугрупповая алгебра полугруппы $\left\{\langle 1, p\rangle \mid p^{2}=p\right\}$. При $\lambda \in(0,1]$ алгебра $A_{\lambda}$ планшерелева.

2 -алгебра $A_{\lambda}$, разумеется, изоморфна 2-алгебре Гекке $H_{2}(q): q=\lambda^{-1} \in[1, \infty)$, соответствие между образующими задается формулой $u=\bar{\tau}$, см. разд. 3.2 .

Теорема 3. 1. Позитивная 2-алгебра $A_{\lambda}$ допускает строгую дилатацию в инволютивную биалгебру (т.е. вкладьвается как строгий подобгект в инволютивную биалгебру, а именно в групповую алгебру конечной коммутативной группы) тогда и только тогда, когда $\lambda$ есть положительный корень уравнения $z^{2}-(2+\alpha) z+1=0$, где $\alpha$ имеет вид $k \cdot \frac{(s-1)^{2}}{s}, k, s-$ произвольные иелье положительные числа. Рациональные значения $\lambda$ получаются при $k=1 u$

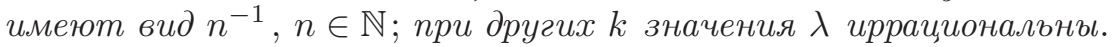

2. Предположим, что $\lambda$ - произвольное рациональное число; тогда $A_{\lambda}$ допускает нестрогую дилатацию в групповую алгебру конечной коммутативной группв.

Например, алгебра $A_{1 / 3}$ допускает нестрогую, но не допускает строгую дилатацию в биалгебру.

В общем случае (см. [5], [9]) $n$-мерная однородная бикоммутативная позитивная 2-алгебра задается матрицей квазихарактеров, т. е. комплексной матрицей, обладающей следующим свойством: покоординатное произведение любых двух строк (столбцов) есть выпуклая комбинация строк (столбцов) и все координаты первой строки и первого столбца равны единице. Для групповой биалгебры коммутативной группы это матрица характеров, для алгебры $A_{\lambda}$ эта матрица имеет вид $\left(\begin{array}{cc}1 & 1 \\ 1 & -\lambda\end{array}\right), \lambda \in[0,1]$.

Проблема нестрогих вложений для бикоммутативных позитивных 2-алгебр в бикоммутативные биалгебры сводится к тому, можно ли матрицу квазихарактеров представить как фактор матрицы характеров некоторой коммутативной группы (или инверсной полугруппы).

\section{ЛитеРАТУРА}

[1] Н. Бурбаки, Топологические векторные пространства, ИЛ, М., 1959.

[2] Э. Баннаи, Е. Ито, Алгебраическая комбинаторика. Схемы отношений, Мир, М., 1987.

[3] В. М. Бухштабер, А. М. Вершик, С. А. Евдокимов, И. Н. Пономаренко, Комбинаторные алгебры и многозначные инволютивные группь, Функц. анализ и его прил., 30:3 (1996), 12-18.

[4] Л. Вайнерман, О работах М. Г. Крейна по теории представлений и гармоническому анализу на топологических группах, Укр. матем. ж., 46:3 (1994), 198-211.

[5] А. М. Вершик, Геометрическая теория состояний, граница фон Неймана, двойственность $C^{*}$-алгебр, Зап. научн. семин. ЛОМИ, 29 (1972), 147-154.

[6] А. М. Вершик, С. А. Евдокимов, И. Н. Пономаренко, Алгебры в планшерелевой двойственности и алгебрачческая комбинаторика, Функц. анализ и его прил., 31:4 (1997), 34-46.

[7] С. А. Евдокимов, Шуровость и отделимость ассоциативных схем, Дисс. д.ф.м.н., СПбГУ, 2005.

[8] К. Кассель, Квантовые группы, Фазис, М., 1999. 
[9] С. В. Керов, Двойственность конечномерных алгебр, Вестник ЛГУ, сер матем., 1974, № 7, 23-29.

[10] С. В. Керов, Двойственность $C^{*}$-алгебр и ее примеры в теории представлений гpynn, Дисс. к.ф.-м.н., ЛГУ, 1974.

[11] А. Клиффорд, Д. Престон, Алгебраическая теория полугрупn, т. 1, Мир, М., 1972.

[12] А. Н. Колмогоров, Собрание сочинений, т. 3, 2003.

[13] М. Г. Крейн, О положительных функиионалах на почти периодических функииях, Докл. АН СССР, 30:1 (1941), 9-12.

[14] М. Г. Крейн, Принцип двойственности для бикомпактной группъ и квадратной блок-алгебры, Докл. АН СССР, 69:6 (1949), 725-728.

[15] М. Г. Крейн, Эрмитово-положительные ядра на однородных пространствах, часть 1, Укр. матем. ж., 1:4 (1949), 64-98.

[16] М. Г. Крейн, Эрмитово-положительные ядра на однородных пространствах, часть 2, Укр. матем. ж., 2:1 (1950), 10-59.

[17] В. А. Оганесян, О полупростоте системной алгебры, Докл. АН Арм. ССР, 21 (1955), 145-147.

[18] Б. Секефальви-Надь, Ч. Фояш, Гармонический анализ операторов в гилъбертовом пространстве, Мир, М., 1970.

[19] S. Bochner, On a theorem of Tannaka and Krein, Ann. of Math., 43 (1942), 56-58.

[20] G. I. Olshansky, Unitary representations of the infinite symmetric group: a semigroup approach, in: Representation of Lie groups and Lie algebras, Akad. Kiado, Budapest, 1985, 181-197..

[21] V. M. Buchstaber, n-valued groups: theory and applications, Moscow Math. J., 6:1 (2006), 57-84.

[22] P. Deligne, Catégories tannakiennes, in: Progr. Math., vol. 87, Birkhäuser, Boston, MA, 1990, 111-195.

[23] A. Joyal, R. Street, An introduction to Tannaka duality and quantum groups, in: Lecture Notes in Math., vol. 1448, Springer-Verlag, Berlin, 1991, 413-492.

[24] D. G. Higman, Invariant relations, coherent configurations, generalized polygons, in: Combinatorics (Proc. Advanced Study Inst., Breukelen, 1974), Part 3: Combinatorial Group Theory, Math. Centre Tracts, No. 57, Math. Centrum, Amsterdam, 1974, 2743.

[25] A. Massoud, Tannaka-Krein duality for compact groups, http://arxiv.org/math.0A/ 0308259.

[26] N. Saavedra Rivano, Catégories tannakienns, Lecture Notes in Math., vol. 265, Springer-Verlag, Berlin-New York, 1972.

[27] L. Scott, A condition on Higman parameters, Notices Amer. Math. Soc., 701 (1972), $20-45$.

[28] L. Scott, Modular permutation representations, Trans. Amer. Math. Soc., 175 (1973), $101-121$.

[29] T. Tannaka, Über den Dualitätssatz der nichtkommutativen topologishen Gruppen, Tohoku Math. J., 45 (1938), 1-12.

[30] A. Van Daele, Quantum groups with invariant integrals, Proc. Nat. Acad. Sci. USA, 97:2 (2000), 541-546.

[31] A. Vershik, Polymorphisms, Markov processes, and quasi-similarity, Discrete Contin. Dyn. Syst., 13:5 (2005), 1305-1324.

[32] А. Вершик, Двойственность Крейна, позитивные 2-алгебры и дилатация коумножений, Препринт ПОМИ № 1, 2007.

С.-Петербургское отделение Математического института им. В. А. Стеклова

e-mail: vershik@pdmi.ras.ru

Поступило в редакцию 7 ноября 2006 г. 